\title{
ASOCIACIÓN ENTRE CARACTERÍSTICAS DE LAS CALCIFICACIONES MAMOGRÁFICAS Y NEOPLASIA MALIGNA EN UN HOSPITAL NACIONAL, 2017
}

\author{
ASSOCIATION BETWEEN CHARACTERISTICS OF MAMMOGRAPHIC CALCIFICATIONS AND MALIGINE \\ NEOPLASIA IN A NATIONAL HOSPITAL - 2017 \\ Víctor Arteaga-Huanca',a, María Loo ${ }^{1,2, b}$, Haylin Looc, Lucy Cedillo-Ramirez
}

\begin{abstract}
RESUMEN
Introducción: La presencia de calcificaciones puede ser útil para el diagnóstico de neoplasia de mama. Objetivo: Determinar la asociación entre las características de las calcificaciones mamográficas y neoplasia maligna. Métodos: Estudio observacional, analítico, retrospectivo de casos y controles. Desarrollado en la Unidad de Patología Mamaria de ginecología. Se incluyeron como casos a pacientes mujeres con mamografía sugerente de neoplasia maligna con biopsia positiva a malignidad y como controles a 67 pacientes sin evidencia histológica de neoplasia. Resultados: La edad media de los casos fue de 54,12 años, en los controles 50,34. El 50,9\%(59) son mayores o iguales a 50 años, OR 2,399 IC 95\% (1,125$5,114) \mathrm{p}=0,022$, de estos el 52,5\% (31) tenían cáncer de mama. El 9,5\%(11) presentaron calcificaciones sospechosas de malignidad, las más frecuentes, la amorfa y la fina pleomórfica. El 37,1\% (43) de los casos totales correspondieron a la categoría BI-RADS 0, el 6,9\% (8) a BI-RADS 1, el 8,6\% (10) a BI-RADS 2, el $7,8 \%$ (9) a BI-RADS 3, el 28,4\% (33)a BI-RADS 4, el 8,6\% (10) a BI-RADS 5 y el 2,6\% (3) a BI-RADS 6. El $72,7 \%$ (8) de pacientes con calcificaciones sospechosas de malignidad presentó cáncer de mama. En el análisis bivariado se obtuvo un OR de 4,163. IC 95\% $(1,043-16,606) p=0,031$. En el análisis multivariado la asociación permaneció inalterada independientemente de la edad y BI-RADS. Conclusión: La edad mayor o igual de 50 años y las calcificaciones mamográficas sospechosas de malignidad son factores asociados para el cáncer de mama.
\end{abstract}

Palabras clave: Cáncer de mama; Mamografía; Neoplasia maligna (fuente: DeCS BIREME).

\begin{abstract}
Introduction: The presence of calcifications may be useful for the diagnosis of breast neoplasia. Objective: To determine the association between the characteristics of mammographic calcifications and malignant neoplasia. Methods: Observational, analytical, retrospective of cases and controls. Developed in the Gynecology Breast Pathology Unit. Female patients with mammogram suggestive of malignant neoplasm with a positive malignancy biopsy were included as cases, and 67 patients without histological evidence of malignancy as controls. Results: The average age of the cases was 54.12 years, in the controls $50.34 .50, .9 \%$ (59) are older than or equal to 50 years, OR $2.39995 \% \mathrm{Cl}(1.125-5.114) \mathrm{p}=0.022$, of these $52.5 \%$ (31) had breast cancer. $9.5 \%$ (11) had calcifications suspicious of malignancy, the most frequent, the amorphous and the pleomorphic fine. $37.1 \%$ (43) of the total cases have the BI-RADS 0 category, 6.9\% (8) BI-RADS 1, 8.6\% (10) BI-RADS 2, $7.8 \%$ (9) BI-RADS 3, 28.4\% (33) BI-RADS 4, 8.6\% (10) BI-RADS 5 and 2.6\% (3) BI-RADS 6. $72.7 \%$ (8) of patients with calcifications suspected of malignancy presented breast cancer. In the bivariate analysis, an OR of 4.163 was obtained. $95 \% \mathrm{Cl}(1.043-16.606) \mathrm{p}=0.031$. In the multivariate analysis the association remained unchanged regardless of age and BI-RADS. Conclusion: Age 50 years or older and mammographic calcifications suspected of malignancy are associated factors for breast cancer.
\end{abstract}

Key words: Breast cancer; Mammography; Malignant neoplasm (source: MeSH NLM).

1 Universidad Ricardo Palma, Lima-Perú.

2 Unidad de Cardiología Pediátrica. Hospital Nacional Edgardo Rebagliati Martins, Lima-Perú.

a Estudiante de medicina.

${ }^{\mathrm{b}}$ Médico pediatra.

c Médico ocupacional.

Citar como: Víctor Arteaga-Huanca, María Loo, Haylin Loo. Asociación entre características de las calcificaciones mamográficas y neoplasia maligna en un hospital nacional, 2017. Rev. Fac. Med. Hum. Enero 20120; 20(1):70-75. DOI 10.25176/RFMH.v20i1.2548 


\section{INTRODUCCIÓN}

El cáncer de mama es el cáncer más frecuente en mujeres a nivel mundial ${ }^{(1,2)}$. En el Perú, el número de casos ocupa el segundo lugar en frecuencia entre las neoplasias reportadas ${ }^{(3)}$.

La guía de cáncer de mama de la National Comprehensive Cancer Network (NCCN), establece que la mamografía y el examen físico son las herramientas que permiten el tamizaje del cáncer de mama, es decir; la identificación de la entidad nosológica(4). La ejecución del tamizaje detecta precozmente los casos de cáncer de mama y hace posible la curación de esta.

La labor del médico radiólogo recae en la identificación de las características mamográficas que tienen valor predictivo positivo a neoplasia maligna y a traducir ese porcentaje a una escala internacional llamada Breast Imaging Reporting and Data System (BI-RADS).

Los países industrializados disminuyen la tasa de mortalidad de cáncer de mama en 2,2\% por año al aplicar el tamizaje y dar tratamiento oportuno ${ }^{(4)}$. Sin embargo, en el Perú, la falta de equipamiento especializado y la poca cultura de prevención no permiten que se establezca un programa tamizaje nacional efectivo, lo cual implica que el cáncer se detecte en estadios tardíos, disminuyendo su probabilidad de curación ${ }^{(5)}$.

El propósito de este trabajo es generar conocimiento para que el apoyo imagenológico permita la identificación de lesiones con valor predictivo positivo a neoplasia maligna en la población peruana.

\section{MÉTODOS}

Se realizó un estudio observacional, analítico, retrospectivo de casos y controles de pacientes mujeres con mamografía y diagnóstico anatomopatológico atendidas en la Unidad de Patología Mamaria del hospital Hipólito Unanue en el año 2017. Se revisaron las historias clínicas de 46 pacientes mujeres (casos) con mamografía sugerente a neoplasia maligna con biopsia positiva a malignidad y 67 (controles) con mamografía sugerente a neoplasia maligna con biopsia negativa a malignidad. Los casos fueron definidos como aquellos pacientes con diagnóstico de cáncer de mama; mientras que los controles fueron aquellas con diagnóstico no correspondiente a malignidad. Fueron evaluados 4 variables potencialmente asociadas: edad de riesgo, calcificaciones sospechosas de malignidad, composición de la mama y categorías BI-RADS.

Las variables numéricas se presentaron como media o mediana y desviación estándar o rango intercuartílico de acuerdo con su distribución. Las variables categóricas fueron presentadas como frecuencias y porcentajes. Se obtuvieron los Odds ratio crudos y ajustados utilizando regresión logística múltiple para evaluar la asociación del tipo de calcificaciones con la presencia de neoplasia demostrada histológicamente.

\section{RESULTADOS}

Se incluyó un total de 116 pacientes que contaron con mamografía y reporte anatomopatológico realizados en la hospitalización de Hipólito Unanue durante el año 2017. Del total de la población, 57,2\%(67) no presentaron cáncer de mama y $42,2 \%$ (49) fueron diagnosticadas de cáncer de mama, de este grupo el $49,1 \%$ (57) fueron menores de 50 años y 50,9\%(59) de igual edad o mayor a 50 años. Las calcificaciones sospechosas de malignidad estuvieron presentes en el 9,5\% (11) de pacientes.

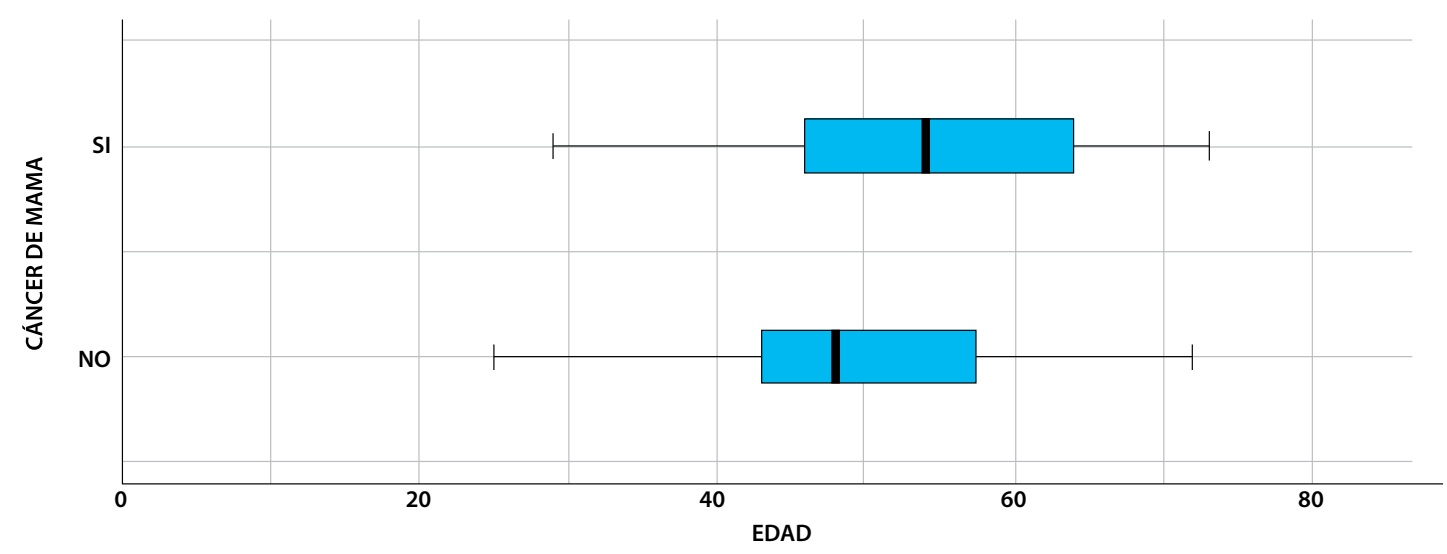

Figura 1. Distribución por edad según cáncer de mama en pacientes de la Unidad de Patología Mamaria. 
En la figura 1, se observa dos cajas. La caja de los pacientes con cáncer de mama es más variable que la de los pacientes sin cáncer de mama. Ambas cajas y bigotes son asimétricas a la derecha. Los valores de edad mínimos y máximos de los pacientes con cáncer son respectivamente: 29 y 73 . La mediana de edad de estas pacientes fue de 54 años con un rango intercuartil de 45,5 a 64 años. Para las pacientes sin cáncer, la edad fluctuó entre 25 y 72 años. La mediana de edad fue de 50 años con un rango intercuartil de 43 a 58 años.
El 37,1\% (43) de los casos totales poseen la categoría BI-RADS 0, el 6,9\% (8) BI-RADS 1, el 8,6\% (10) BI-RADS 2 , el 7,8\% (9) BI-RADS 3, el 28,4\% (33) BI-RADS 4, el $8,6 \%$ (10) BI-RADS 5 y el 2,6\% (3) BI-RADS 6.

La composición de la mama fue: casi en su totalidad tejido adiposo 8,6\% (10), con sectores dispersos de densidad fibroglandular 39,7\% (46), heterogéneamente densa $37,9 \%(44)$ y muy densa $13,8 \%$ (16).

Tabla 1. Características de los pacientes con calcificaciones mamográficas sospechosas de malignidad.

\begin{tabular}{|c|c|c|c|c|c|}
\hline Edad & Grado BIR-RADS & \multicolumn{2}{|c|}{ Diagnóstico } & \multicolumn{2}{|c|}{ Calcificaciones } \\
\hline 58 & $4 c$ & \multicolumn{2}{|c|}{ Carcinoma ductal infiltrante } & \multicolumn{2}{|c|}{ Finas pleomórficas } \\
\hline 55 & $4 b$ & \multicolumn{2}{|c|}{ Carcinoma ductal infiltrante y carcinoma in situ } & \multicolumn{2}{|c|}{ Amorfas agrupadas } \\
\hline 54 & $4 c$ & \multicolumn{2}{|c|}{ Carcinoma ductal infiltrante y carcinoma in situ } & \multicolumn{2}{|c|}{ Finas pleomórficas } \\
\hline 62 & 5 & \multicolumn{2}{|c|}{ Carcinoma ductal infiltrante } & \multicolumn{2}{|c|}{ Finas pleomórficas } \\
\hline 50 & 5 & \multicolumn{2}{|c|}{ Carcinoma ductal infiltrante } & \multicolumn{2}{|c|}{$\begin{array}{l}\text { Finas ramificadas y finas } \\
\text { pleomórficas }\end{array}$} \\
\hline 42 & $4 c$ & \multicolumn{2}{|c|}{ Carcinoma lobulillar infiltrante } & \multicolumn{2}{|l|}{ Amorfas } \\
\hline 72 & 5 & \multicolumn{2}{|c|}{ Carcinoma ductal infiltrante y carcinoma in situ } & \multicolumn{2}{|l|}{ Amorfas } \\
\hline 45 & 0 & \multicolumn{2}{|l|}{ Carcinoma in situ } & \multicolumn{2}{|c|}{ Gruesas heterogéneas } \\
\hline 49 & $4 a$ & \multicolumn{2}{|c|}{ Mastopatia fibroquistica } & \multicolumn{2}{|c|}{$\begin{array}{l}\text { Microcalcificaciones } \\
\text { sospechosas }\end{array}$} \\
\hline 47 & $4 b$ & \multicolumn{2}{|l|}{ Fibroadenoma } & \multicolumn{2}{|c|}{ Finas pleomórficas } \\
\hline 43 & 5 & \multicolumn{2}{|l|}{ Fibroadenoma } & \multicolumn{2}{|c|}{ Amorfas agrupadas } \\
\hline \multicolumn{6}{|c|}{$\begin{array}{ll}\text { Se detectaron } 8 \text { casos de cáncer de mama con } & \text { lobulillar infiltrant } \\
\text { calcificaciones sospechosas y } 3 \text { controles detectados } & \text { encontradas fuero } \\
\text { con calcificaciones sospechosas sin neoplasia maligna. } & \text { finas ramificadas, fi } \\
\text { Las estirpes histológicas encontradas son carcinoma } & \text { En relación a los BI } \\
\text { ductal infiltrante, carcinoma in situ y el carcinoma } & 4 \mathrm{~b}, 4 \text { c y } 5 .\end{array}$} \\
\hline \multicolumn{2}{|c|}{ Calcificación sospechosa } & $\begin{array}{l}\text { Carcinoma ductal } \\
\text { infiltrante }\end{array}$ & $\begin{array}{l}\text { Carcinoma lobulillar } \\
\text { infiltrante }\end{array}$ & $\begin{array}{l}\text { Carcinoma in } \\
\text { situ }\end{array}$ & Total \\
\hline \multicolumn{2}{|c|}{ Amorfa } & 2 & 1 & 0 & $3(37,5 \%)$ \\
\hline \multicolumn{2}{|c|}{ Gruesa heterogénea } & 0 & 0 & 1 & $1(12,5 \%)$ \\
\hline \multicolumn{2}{|c|}{ Finas pleomórficas } & 3 & 0 & 0 & $3(37,5 \%)$ \\
\hline \multicolumn{2}{|c|}{ Fina ramificada } & 1 & 0 & 0 & $1(12,5 \%)$ \\
\hline \multicolumn{2}{|l|}{ Total } & $6(75 \%)$ & $1(15 \%)$ & $1(15 \%)$ & $8(100 \%)$ \\
\hline
\end{tabular}

Las calcificaciones sospechosas más frecuentes en finas pleomorficas, ambas con $37,5 \%$ de los casos. neoplasia maligna de mama fueron, la amorfa y las 
Tabla 3. Asociación entre edad de riesgo y cáncer de mama.

\begin{tabular}{|c|c|c|c|c|c|}
\hline Edad de riesgo & Cáncer de mama & No cáncer de mama & Valor de p & OR & IC $95 \%$ \\
\hline Menores $(68,4 \%)$ de 50 años & $18(31,6 \%)$ & $\begin{array}{l}\text { Biomédica } \\
39(68,4 \%)\end{array}$ & 0,022 & 2,40 & $1,1-5,1$ \\
\hline Mayores $(47,5 \%)$ de 50 años & $31(52,5 \%)$ & $\begin{array}{l}\text { Biomédica } \\
28(47,5 \%)\end{array}$ & & & \\
\hline
\end{tabular}

En mayores de 50 años el 52,5\% (31) tenían cáncer de El Odds Ratio fue de 2,40 IC 95\% $(1,13-5,11)$ con un mama y el $47,5 \%$ (28) no eran portadoras de cáncer. valor de $\mathrm{p}=0,022$.

Tabla 4. Asociación entre calcificaciones sospechosas y cáncer de mama.

\begin{tabular}{cccccc}
$\begin{array}{c}\text { Calcificaciones sospechosas a } \\
\text { malignidad }\end{array}$ & $\begin{array}{c}\text { Cáncer de } \\
\text { mama }\end{array}$ & No cáncer de mama & Valor de p & OR & IC 95\% \\
No $(61 \%)$ & $41(39 \%)$ & $\begin{array}{c}\text { Biomédica } \\
64(61 \%)\end{array}$ & 0,031 & 4,16 & $1,04-16,60$ \\
Si $(27,3 \%)$ & $8(72,7 \%)$ & $\begin{array}{c}\text { Biomédica } \\
3(27,3 \%)\end{array}$ & & & \\
\hline
\end{tabular}

El $72,7 \%$ (8) de pacientes con calcificaciones obtuvo un Odds Ratio de 4,16. IC 95\% $(1,04-16,61)$ y sospechosas de malignidad presentaron cáncer de un valor de $p=0,031$, encontrándose una asociación mama y el 27,3\% (3) no tuvieron cáncer de mama. Se significativa.

Tabla 5. Asociación entre el grado de Bi-Rads y cáncer de mama.

\begin{tabular}{|cccccc|}
\hline Grado de BI-RADS & Cáncer de mama & No cáncer de mama & Valor de p & OR & IC 95\% \\
\hline Bajo riesgo $(70,4 \%)$ & $8(29,6 \%)$ & $\begin{array}{l}\text { Biomédica } \\
19(70,4 \%)\end{array}$ & 0,041 & 2,83 & $1,03-7,76$ \\
Alto riesgo $(45,7 \%)$ & $25(54,3 \%)$ & $\begin{array}{l}\text { Biomédica } \\
21(45,7 \%)\end{array}$ & & & \\
\hline
\end{tabular}

De las pacientes con BI-RADS de alto riesgo (categorías 4,5 y 6) el 54,3\% (25) presentaron cáncer de mama en comparación con el $29,6 \%$ de las pacientes con bajo riesgo (categorías 1,2 y 3). Se obtuvo un Odds Ratio de 2,83 IC 95\% (1,03 - 7.76) y un valor de $p=0,041$, encontrándose una asociación significativa.

\section{DISCUSIÓN}

El cáncer de mama es el cáncer más frecuente en mujeres a nivel mundial(1). En el Perú es un problema de salud pública dado que tiene una elevada carga de enfermedad y además genera costos elevados para su tratamiento6. Por esa razón, al conocer los factores que permiten precisar su diagnóstico es de vital importancia.

En nuestro estudio encontramos que la edad media de las pacientes con cáncer de mama fue de 54 años. Las pacientes con 50 años o más con cáncer de mama representaron el $63.3 \%$ del total. Esto avala y da soporte a las recomendaciones de la Sociedad Americana de Cáncer para el cribado de cáncer de mama. Tal entidad sugiere que las mujeres entre 45 y 54 años deben realizarse el examen mamográfico
En el análisis multivariado el riesgo de BI-RADS presentó un OR de 1,21 IC 95\%(0,72-2,02) y valor de $\mathrm{p}=0,469$, siendo no significativo. La edad mayor a 50 años presentó un OR de 2,54 IC 95\% (1,16-5,56), $\mathrm{p}=0,020$, mientras que las calcificaciones sospechosas de malignidad presentaron un OR de $4,44(1,07-18,37)$ y un valor $p=0,040$.

Anualmente $^{(7)}$. La incidencia en mujeres de este mal oncológico en el Perú es de 40 por cien mil habitantes; sin embargo, la incidencia entre los 45 y los 54 años es de 95 por cien mil(8).

La asociación entre edad y cáncer de mama es ampliamente conocida y la tendencia es que a mayor edad, mayor cáncer ${ }^{(8)}$. En relación a esta variable, en el estudio se encontró que el 52,5\% de mayores de 50 años se encontraba dentro de los casos de cáncer de mama, con lo cual se obtuvo un Odds Ratio de 2,40 IC $95 \%(1,13-5,11)$ y un valor $p=0,022(<0,05)$. Esto contrasta con la tendencia demostrada por $\mathrm{S}$. Eve Singletary(9) en "Rating the Risk Factors for Breast Cancer", donde la incidencia es extremadamente baja en menores de 30 años (incidencia menos de 25 por 100 000). Pero que aumenta linealmente hasta la edad 
de 80 años. El riesgo relativo de las mujeres mayores de 65 años comparado con las menores de 65 años es de 5,8 en dicho estudio.

Con respecto al grado de BI-RADS, en el presente estudio se encontró que el $54,3 \%$ de pacientes con $\mathrm{BI}$ RADSS de alto riesgo (categorías 4,5 y 6 ) se encuentran dentro de los casos de cáncer de mama. Se obtuvo un Odds Ratio de 2,827 (IC 95\% (1,03 - 7,758) y un valor $p=0,041$. Lo que revela que tener un BI-RADS de alto riesgo (categorías 4, 5 y 6) confiere un riesgo de 2,8 veces en relación a tener un BI-RADS de bajo riesgo (categorías 1, 2, 3), no se consideró el grado 0 pues por definición requiere de otra prueba mamográfica más especializada (amplificación) o de una ecografía para catalogarla adecuadamente. La clasificación BIRADS es desarrollada por el Colegio Americano de Radiología y como se mencionó, es la herramienta de vigente uso para informar las mamografías ${ }^{(10)}$. Su importancia radica en que mediante la descripción de lesiones se predice la presencia de malignidad en el paciente, siendo una herramienta muy útil para la indicación de biopsia. En el estudio de Chris Bent y colegas ${ }^{(11)}$, titulado: "The Positive Predictive Value of BI-RADS Microcalcification Descriptors and Final Assessment Categories" se encuentra que el riesgo de malignidad de acuerdo a las categorías del BI-RADS aumentan progresivamente, la diferencia entre cada categoría ( $p<0,001)$ y cada subcategoría $(p<0,001)$ son estadísticamente significativas, demostrando que el radiólogo puede estratificar satisfactoriamente el potencial de malignidad con el BI-RADS. Los valores predictivos positivos para malignidad de acuerdo al sistema Bi-RADS fueron los siguientes: categoría 2: 0\%, categoría 3: 0\%, categoría 4: 13\%, categoría 4B: 36\%, categoría 4C: 79\%; y categoría 5, 100\% en contraste con nuestro trabajo se coincide en que a mayor BIRADS mayor riesgo de malignidad; sin embargo, por limitantes estadísticas la regresión logística de las categorías y subcategorías del BI-RADS fueron no significantes en el análisis multivariado.

La neoplasia maligna más frecuente en nuestro estudio es el carcinoma ductal infiltrante (75\%), seguido de carcinoma lobulillar infiltrante (15\%) y carcinoma in situ (15\%). Similar a esto, se observa en el resultado del estudio epidemiológico descriptivo realizado desde el año 1987 hasta el año 1999 por Christopher Li y colegas ${ }^{(12)}$, titulado "Trends in Incidence Rates of Invasive Lobular and Ductal Breast Carcinoma", donde se estableció que tipo histológico más frecuente en cáncer de mama es el tipo carcinoma ductal infiltrante $(72,8 \%)$ seguido del carcinoma lobulillar infiltrante $(7,6 \%)$ y de carcinoma ductal y lobulillar infiltrante $(4,7 \%)$ de una población de 190458 casos.
En relación a la composición de la mama, se encontró que aquellas constituidas por sectores dispersos de densidad fibroglandular son los más frecuentes, seguido de las mamas heterogéneamente densas. Ello contrasta con el estudio de Madiha Naseem y colegas $^{(13)}$, en donde se asocia significativamente ( $p=0,031$ ), luego de evaluar a 937 mujeres, las calcificaciones sospechosas de malignidad con densidad mamaria heterogénea a predominio fibroglandular. En nuestro trabajo no se encontró asociación estadística con el cáncer de mama ni con las calcificaciones mamográficas. Se conoce que la densidad de la mama aumentada es un factor de riesgo para el cáncer de mama dado que evita la visualización de hallazgos mamográficos disminuyendo la sensibilidad ${ }^{(14)}$. Las calcificaciones sospechosas de malignidad son agrupadas en este estudio y se demuestra que tienen asociación significativa con neoplasia maligna. Se observa que el $72,7 \%$ de pacientes con calcificaciones sospechosas de malignidad se encuentran dentro de los casos de cáncer de mama. Con un valor $p=0,031$ IC 95\% (1,04 - 16,61).Tener este tipo de calcificaciones representa un riesgo alrededor de 4 veces mayor en comparación al grupo que no las posee. Las características de las calcificaciones son descritas según la quinta edición del BI-RADS(15); sin embargo, uno de los controles fue descrito como calcificaciones sospechosas, termino parcialmente correcto, pues el hecho que sea sospechosa no significa que no debe describirse el tipo de calcificación. A su vez, la correlación de la presencia de algún tipo de calcificación sospechosa condiciona un grado de BI-RADS que va del 4 al 5 , no siendo coherente en el sistema BI-RADS definir un caso con una calcificación sospechosa (gruesa heterogénea) como BI-RADS 0 , evento que sucede en uno de los controles de este estudio ${ }^{(16)}$. Las calcificaciones asociadas a malignidad encontradas fueron por orden de frecuencia: finas pleomórficas $(37,5 \%)$, amorfas $(37,5 \%)$, finas ramificadas $(12,5 \%)$ y gruesas heterogéneas (12,5\%). En el estudio, titulado "Mammographic microcalcifications and breast cancer tumorigenesis: a radiologic-pathologic analysis" asocian la morfología con riesgo de malignidad ( $p<0,001$ ), siendo la fina ramificada la que tiene mayor riesgo de malignidad con $70 \%$, seguida de finapleomórfica (28\%), heterogénea gruesa (20\%), y amorfa $(20 \%)^{(11)}$.

\section{CONCLUSIÓN}

Las calcificaciones mamográficas sospechosas de malignidad fueron un factor fuertemente asociado a la presencia de cáncer de mama independientemente de la edad y clasificación de BI-RADS. 
Contribuciones de autoría: Los autores participaron en la concepción, redacción y aprobación final del artículo original, además VAH en la recolección de datos. Financiamiento: Autofinanciado.

Correspondencia: Víctor Gabriel Arteaga Huanca.

Dirección: Avenida Perú 1680 San Martín de Porres, Lima-Perú.

Teléfono: +993819417

Correo:victor_arteaga92@hotmail.com
Conflicto de interés: Los autores declaran no tener conflicto de interés en la publicación de este artículo.

Recibido: 20 de julio del 2019

Aprobado: 10 de noviembre del 2019

\section{REFERENCIAS BIBLIOGRÁFICAS}

1. Organización Mundial de la Salud. Nota descriptiva. Cáncer. Septiembre 2018. Disponible en: https://www.who.int/news-room/fact-sheets/detail/cancer

2. Instituto Nacional de Enfermedades Neoplásicas. Casos nuevos de cánce registrados en INEN, periodo 2000-2016. Disponible en: https://portal.inen.sld.pe/ wp-content/uploads/2018/06/INEN-CASOS-NUEVOS-2000-2016.pdf

3. Warner E. Clinical practice. Breast-Cancer screening. N Engl Med. 2011 365(11):1025-32. DOI: 10.1056/NEJMcp1101540

4. Instituto Nacional de Estadística e Informática. Programa de prevención y control del cáncer. Perú, enfermedades no trasmisibles y transmisibles. Lima-Perú. 2016. p. 83 - 94. Disponible en: www.inei.gob.pe/media/MenuRecursivo/publicaciones digitales/Est/Lib1432/cap02.pdf

5. Poquioma E. Situación del cáncer de mama en el Perú. Departamento de Epidemiologia y Estadística del INEN. 2008. Disponible en: http://bvsper.paho.org/ videosdigitales/matedu/20111205_Cancer_Mama_Epi.pdf?ua=1

6. Oeffinger KC, Fontham ETH, Etzioni R, et al. Breast Cancer Screening for Women at Average Risk: 2015 Guideline Update from the American Cancer Society. JAMA. 2015;314(15):1599-1614. doi:10.1001/jama.2015.12783

7. World Health Organization. Breast Global Cancer Observatory. Globocan 2018 Disponible en: https://gco.iarc.fr/today/data/factshetts/cancer/20-Breast-factsheet.pdf

8. Singletary S. E. Rating the Risk Factors for Breast Cancer. Annals of Surgery. 2003; 237(4): 474-82. Disponible en: https://www.ncbi.nlm.nih.gov/pmc/articles/ PMC1514477/pdf/20030400s00007p474.pdf
9. American College of Radiology. BI-RADS. Sistema de informes y registro de datos de estudios por imágenes de mamas.5ta. ed. Journal;2018. Disponible en:https://www.edicionesjournal.com/Papel+Digital/9789871981861/BIRADS\%C2\%AE+5\%C2\%AA+Ed++-+Sistema+de+informes+y+registro+de+datos+ de+imagen+de+mama

10. Chris K, Lawrence W, Carl Ji, James W. The Positive Predictive Value of BI-RADS Microcalcification Descriptors and Final Assessment Categories. American Journa of Radiology, may 2010; 194(5): 1378-83. DOI: 10.2214/AJR.09.3423.

11. $\mathrm{Li} \mathrm{Cl}$, Anderson BO, Daling JR, Moe RE: Trends in incidence rates of invasive lobular and ductal breast carcinoma. JAMA 2003; 289(11):1421-24. DOI: 10.1001/ jama.289.11.1421

12. Naseem M, Murray J, Hilton J F, et al. Mammographic microcalcifications and breast cancer tumorigenesis: a radiologic-pathologic analysis. BMC Cancer.2015 15:307. DOI: 10.1186/s12885-015-1312-z.

13. Kennedy G, Markert M, Alexander JR, Avisar E. Predictie value of BI-RADS classification for breast imaging in women under age 50 . Breast Cancer Research and Treatment. 2011; 130(3):819-23. doi: 10.1007/s10549-011-1669-x.

14. Spak D.A. Plaxco J.S. Santiago L. Drydenb M.J. Dogan B.E. BI-RADS fifth edition: A summary of changes.Diagn Intery Imaging. 2017;98(3): 179-90. DOI: 10.1016/j. diii.2017.01.001

15. Rominger $\mathrm{M}$, Wisgickl $\mathrm{C}$ and Timmesfeld $\mathrm{N}$. Breast microcalcifications as type descriptors to stratify risk of malignancy: a systematic review and meta-analysis of 10665 cases with special focus on round/punctate microcalcifications. RöFo. 2012·184(12):1144-52. DOI: $10.1055 /$ s-0032-1313102.

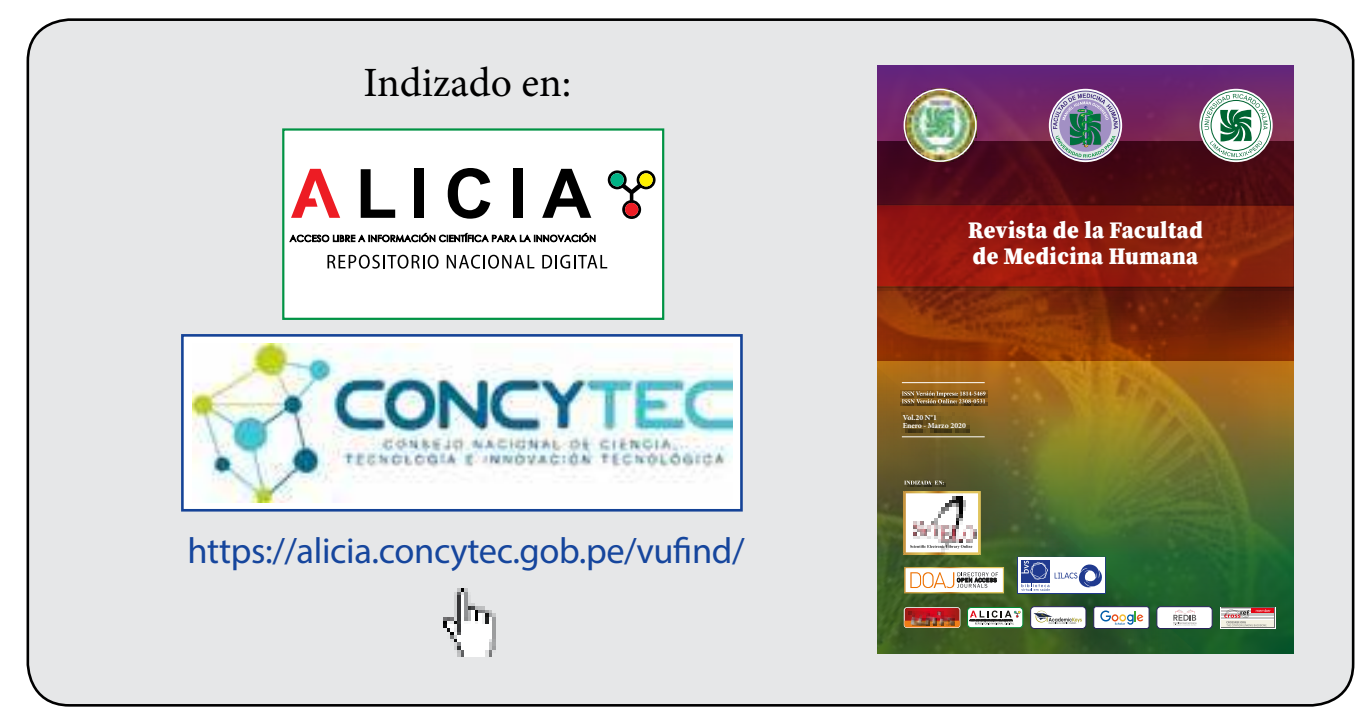

